\title{
NUMERICAL SIMULATIONS OF SEDIMENT TRANSPORT INDUCED BY THE 2004 INDIAN OCEAN TSUNAMI NEAR KIRINDA PORT IN SRI LANKA
}

\author{
Naoto Kihara and Masafumi Matsuyama ${ }^{1}$
}

\begin{abstract}
Numerical simulations of sediment transport induced by the 2004 Indian Ocean tsunami near Kirinda port in southeastern Sri Lanka is carried out and the relationships between the tsunami-induced flow and sediment transport are discussed. The results show two clear relationships. The first one is that the local scour occurs and a large amount of suspended sediment is generated around the head of breakwaters when the tsunami-induced flow passes through there. The second one is that the suspended sediment is deposited near vortex centers due to a secondary flow.
\end{abstract}

Keywords: Sediment transport; three-dimensional simulation; secondary flow

\section{Introduction}

Large-scale tsunamis cause extensive sediment transport in coastal areas. Tsunami-induced sediment transport causes local scours and depositions around coastal facilities, possibly leading to the loss of their function on occasion. Tomita et al. (2005) reported that extensive erosion was observed around coastal structures and piers in southwest Sri Lanka after the 2004 Indian Ocean tsunami, and their functions were lost even though damage was not observed in the structures themselves.

The velocity of the flow induced by a large-scale tsunami in harbors is very high, 1-20 m / s, and the wave period is longer than $10 \mathrm{~min}$, which is much longer than that of wind waves and much shorter than that of flow rate changes in rivers under flood conditions. Thus, doubt remains on whether the sediment transport models for river flow and coastal wind waves can be directly used to evaluate tsunami-induced sediment transport, and special calibrations may be needed (Takahashi et al., 1999).

To evaluate tsunami-induced topography changes, they have been studied experimentally and numerically over the past decade. Takahashi et al. (1999), Fujii et al. (2009), and Nishihata et al. (2006) developed vertically-averaged two-dimensional numerical models to calculate tsunami-induced topography changes. They carried out numerical simulations and compared their results with those of experimental studies and field surveys, and their numerical results show some agreements with those of experiments. Kihara and Matsuyama (2008, 2010) developed a three-dimensional hydrostatic shallow-water model, C-HYDRO3D. They carried out numerical simulations for comparison with the experimental studies conducted by Fujii et al. (2009) and Sakakiyama et al. (2010) and showed the importance of suspended sediment transport due to secondary flow in the predictions of deposition areas in harbors, which cannot be described by the vertically averaged two-dimensional simulations.

In the present study, numerical simulations of sediment transport induced by the 2004 Indian Ocean tsunami near Kirinda port in southeastern Sri Lanka are carried out, to investigate the characteristics of tsunami-induced sediment transport in actual coastal areas. This case has already been studied by two-dimensional simulations by Nishihata et al. (2006) and Takahashi et al. (2008). Furthermore, we discuss the relationships between the tsunami-induced flow and sediment transport.

\section{Numerical Model and Setup}

The numerical model used in this study is the three-dimensional hydrostatic shallow water model, the C-HYDRO3D. The governing equations of flow are the continuity equation and the momentum equation with hydrostatic approximation. The eddy diffusivity is estimated using the Mellor-Yamada level 2 closure model (Mellor and Yamada, 1982). The sediment transport is calculated by solving conservation equations of the suspended sediment in the water and the sediment on the bed. These equations are transformed into a terrain-following coordinate system. The bed-load transport rate and entrainment rate into the suspended load layer are estimated using Van Rijn formulae (1984 (a), (b)).

Using C-HYDRO3D, we estimate the topography changes near Kirinda port in Sri Lanka induced by the 2004 Indian Ocean Tsunami. The topography near Kirinda port is shown in Fig. 1. In Kirinda port, there are four breakwaters: a main breakwater, a secondary breakwater, an old secondary breakwater, and a sand control wall. Before the 2004 Indian Ocean tsunami, the bed level at the open mouth of Kirinda port tended to rise temporally, and the bed level was higher than the mean water level. However after the tsunami, the bed level declined due to local erosion and became lower than the mean water level (Nishihata et al., 2006).

\footnotetext{
${ }^{1}$ Central Research Institute of Electric Power Industry, 1646 Abiko, Abiko-shi, Chiba, 270-1197, Japan
} 


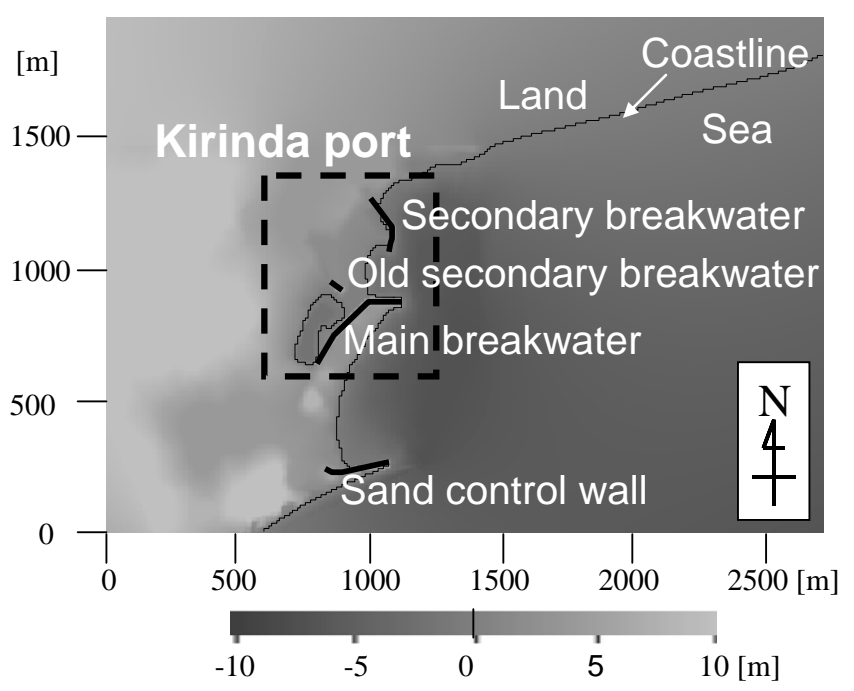

Figure 1. The topography near Kirinda port. Thin solid lines denote coastal lines before the 2004 Indian Ocean Tsunami.


Figure 2. The computational domains.

Fig. 2 shows the computational domains used in this study. To calculate tsunami propagation on the global scale and the tsunami-induced sediment transport in the harbor simultaneously, a nested grid system of eight domains with different resolutions is used. The horizontal grid resolution in the smallest domain (domain 8) is $3.3 \mathrm{~m}$ and that in the largest domain (domain 1) is $7290 \mathrm{~m}$. The grid numbers in the horizontal directions are $450 \times 450$ for domain $1,150 \times 150$ for domain 2, $150 \times 150$ for domain 3, $150 \times 150$ for domain 4, $150 \times 150$ for domain 5, $150 \times 150$ for domain $6,150 \times 150$ for domain 7, and $279 \times 201$ for domain 8 . The number of vertical layers is eight. Since the grid number for domain 1 is very large compared with those of the other domains, a two-dimensional simulation is carried out for domain 1, although three-dimensional simulations are carried out for the other domains. The numerical grid is constructed by interpolating the GEBCO bathymetry data sets for the offshore region. The bathymetry survey data obtained by the Japan International Cooperation Agency are used near Kirinda port. On land, SRTM bathymetry data sets are used. The median diameter of the bed material is $0.12 \mathrm{~mm}$, as reported by Nishihata et al. (2006) and Takahashi et al. (2008).

The accuracy of the tsunami-induced topography changes in Kirinda port mainly depends on the accuracy of the modeling of the behavior of tsunami inundation in that area, which strongly depends on the tsunami source model used. Therefore, in this study, we carry out numerical simulations for two cases. In case O06, the tsunami-source models proposed by Oie et al. (2006) are used, and in case F07, the tsunami-source models proposed by Fujii and Satake (2007) are used. These source models were developed using coastal tide gauge station data and satellite transect records of JASON1, TOPEX/Poseidon, and Envisat. In case O06, the tsunami is generated by inducing water level displacements with 6 subfaults in the initial condition. On the other hand, in case F07, the tsunami is 


\begin{tabular}{|c|c|c|c|}
\hline \multicolumn{4}{|c|}{ Table 1. Tsunami arrival time. Unit is minute. } \\
\hline & Field survey reports & Case O06 & Case F07 \\
\hline Kirinda & 136 (Inoue et al., 2007) & 128 & 139 \\
Yala & $131-136$ (Inoue et al., 2007) & 126 & 137 \\
Hambantota & $131-143$ (Inoue et al., 2007) & 128 & 139 \\
\hline
\end{tabular}

\begin{tabular}{|c|l|c|c|}
\hline \multicolumn{5}{|c|}{ Table 2. Maximum water level. Unit is m. } \\
\hline & Field survey reports & Case O06 & Case F07 \\
\hline Kirinda & $8.7 \quad$ (Inoue et al., 2007) & 7.1 & 5.7 \\
& $8.4 \quad$ (Nishihata et al., 2005) & & \\
& $6.9-9.3$ (Shibayama et al., 2005) & & 5.3 \\
\hline Yala & $5.2 \quad$ (Liu et al., 2005) & 8.6 & 5.2 \\
& $5.7-10.5$ (Inoue et al., 2007) & & \\
\hline Hambantota & $6.9 \quad$ (Liu et al., 2005) & 7.4 & \\
& $10-11$ (Papadopoulos et al., 2006) & & \\
& $7.2-10.9$ (Shibayama et al., 2005) & & \\
\hline
\end{tabular}

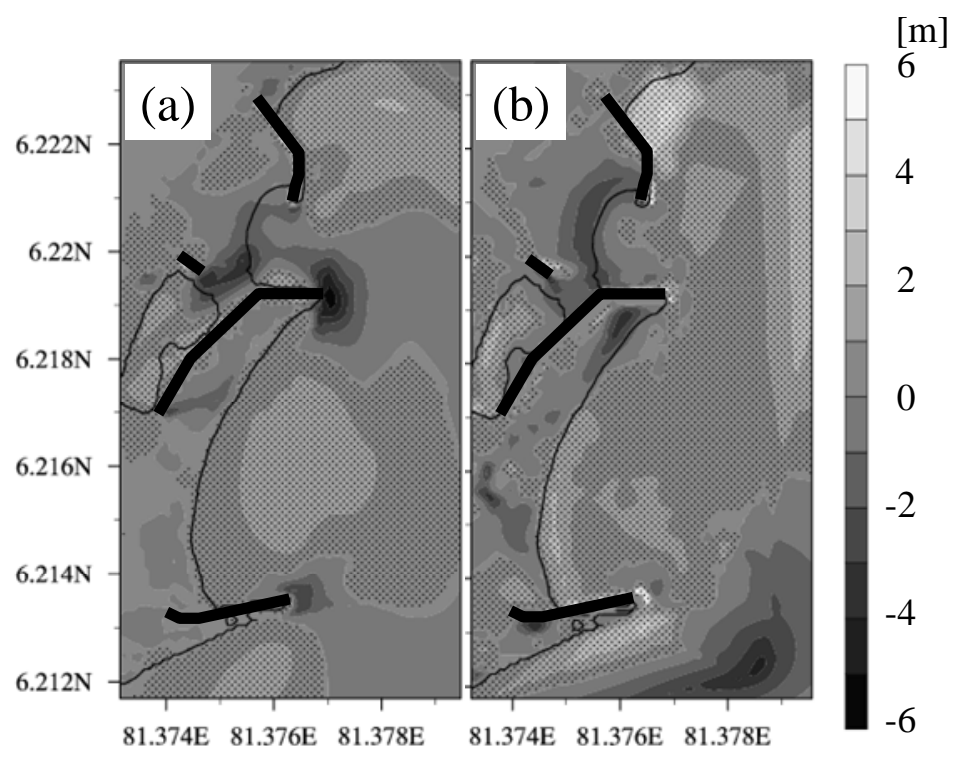

Figure 3. The topography changes by the 2004 Indian Ocean Tsunami. (a) Numerical results, (b) bathymetry survey data. Dot denotes deposition area.

generated by inducing the temporal seabed deformations with 22 subfaults, taking into consideration with the rupture velocity and rise time.

\section{Results and discussion}

In table 1, we show the tsunami arrival times at Kirinda, Yala, and Hambantota obtained by the numerical simulations and those reported by field surveys. Yala is located about $20 \mathrm{~km}$ northeast of Kirinda, and Hambantota is located about $25 \mathrm{~km}$ southwest of Kirinda. From the field survey reports, the tsunami arrival times at those areas were between 131 and 143 minutes after the occurrence of the earthquake. The estimated tsunami arrival times for case 006 were earlier than those of the field survey reports, while those for case F07 were in the range of the field survey values. The reason for the earlier estimates in case $\mathrm{O} 06$ is the tsunami generation method. As mentioned in the previous section, the tsunami is generated by inducing water level displacements under the initial condition in case O06, whereas temporal seabed deformations are used in case F07. Such instantaneous tsunami generations in case O06 would make the tsunami generation time earlier, so that the tsunami arrival times become earlier.

In table 2, we show maximum water levels (MWLs) at Kirinda, Yala, and Hambantota obtained by the numerical simulations and those reported by field surveys. The estimated MWLs in case O06 were in the range of field survey values. On the other hand, in case F07, the estimated MWLs were much 


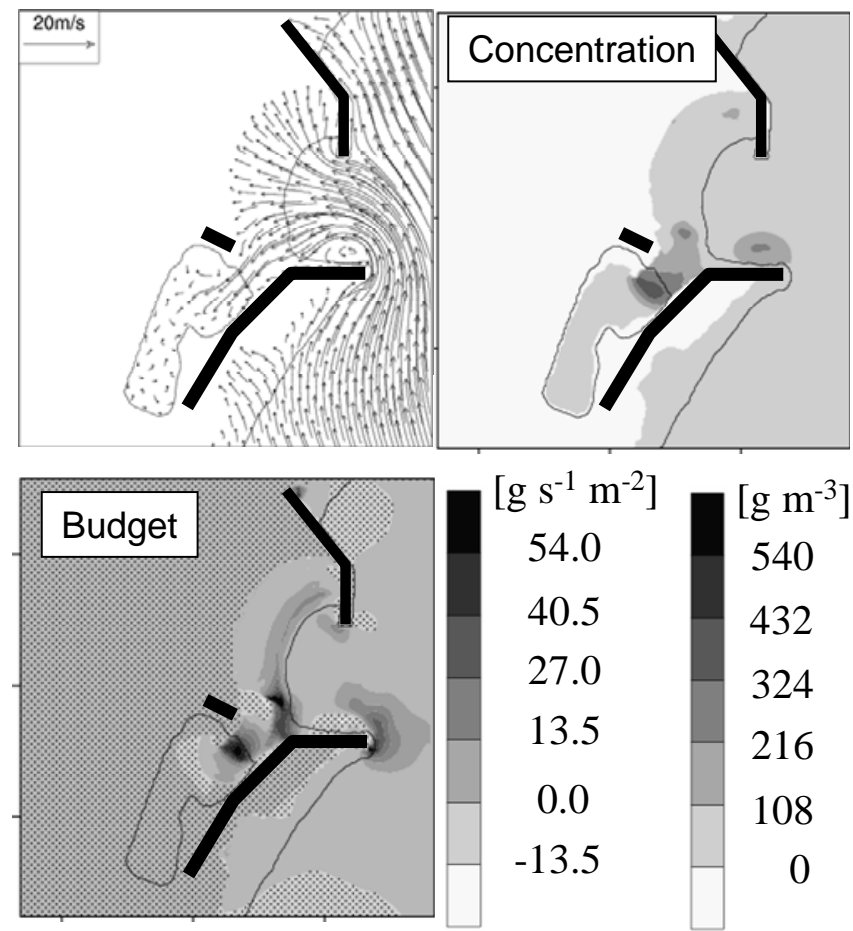

Figure 4. Spatial distributions of the vertically averaged horizontal velocity vectors, the vertically averaged suspended sediment concentrations, and budgets of suspended sediment concentration near the bed with time when the tsunami-induced flow enters into Kirinda port.

lower than those of field surveys. This lower estimate of MWLs would lead to erroneous topography changes. Thus, in the following analyses, we focus on the numerical results of case O06.

We compare topography changes obtained in the present study with those estimated by bathymetry surveys in Fig. 3. The bathymetry surveys were carried out by the Japan International Cooperation Agency about one month before and after the 2004 Indian Ocean tsunami. From the bathymetry survey results, erosion is observed at the open mouth of the port and depositions are observed to the north of the secondary breakwater and the sand control wall. The numerical results are in agreement with the bathymetry survey results in the landward area from the head of the main breakwater, whereas some disagreement is observed in the seaward area from the head of the main breakwater.

To investigate the relationship between the tsunami-induced flow and the tsunami-induced sediment transport, we show spatial distributions of the vertically averaged horizontal velocity vectors, the vertically averaged suspended sediment concentrations, and budgets of suspended sediment concentration near the bed. The budget is the difference between the masses of sediment near the bed entrained in and removed from the water at a given time. Since the suspended load is dominant over the bed load because of the high Shield number flow, erosion occurs at the positive budget areas and deposition occurs at the negative budget areas. Fig. 4 shows those distributions with time when the tsunami-induced flow enters into Kirinda port, and Fig. 5 shows those distributions with time when the tsunami-induced flow inundates the area around Kirinda port. Fast flow is observed at the head of the main breakwater in Fig. 4 (a), and positive budget values are also observed there from Fig. 4 (c), showing that local erosion occurs when the tsunami-induced flow passes through there.

Fig. 5 (a) shows some vortices around Kirinda port, and high suspended sediment concentrations are observed near the centers of the vortices in Fig. 5 (b). The cause of the high suspended sediment concentrations at the vortex centers is due to secondary flow. At areas with flow curvature such as the vortices, the Ekman layer develops near the bed and a secondary flow is generated. This secondary flow transports suspended sediment near the bed toward the vortex center, so that the suspended sediment concentration becomes high near the vortex center.

Negative budget values are also observed at the vortex centers (Fig. 5 (c)), indicating deposition occurs near the vortex centers. This is because the flow velocity is low at the vortex centers and the suspended sediment with a high concentration tends to deposit there. 


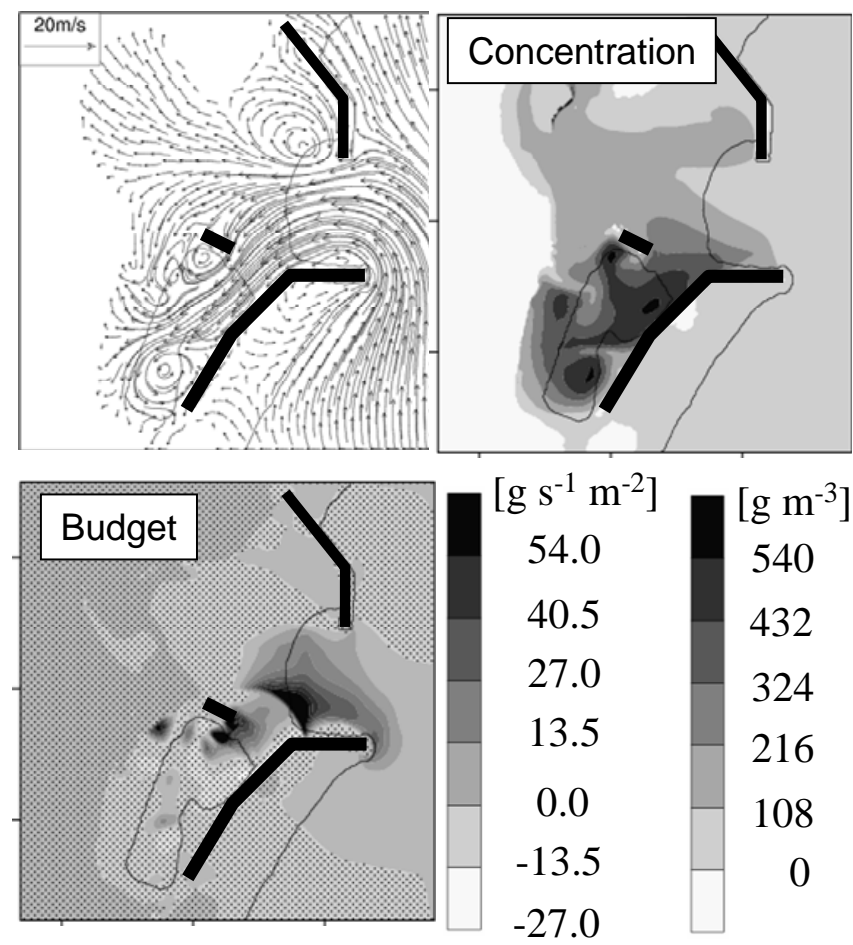

Figure 5. Spatial distributions of the vertically averaged horizontal velocity vectors, the vertically averaged suspended sediment concentrations, and budgets of suspended sediment concentration near the bed with time when the tsunami-induced flow inundates the area around Kirinda port.

\section{Conclusions}

Numerical simulations of sediment transport induced by the 2004 Indian Ocean tsunami near Kirinda port in southeastern Sri Lanka were carried out and the relationships between the tsunamiinduced flow and sediment transport were discussed.

The results showed two clear relationships. The first one is that local scour occurs and a large amount of suspended sediment is generated around the heads of breakwaters when the tsunamiinduced flow passes through there. The second one is that the suspended sediment is deposited near the vortex centers, since the secondary flow transports suspended sediment toward the vortex centers where the velocity is low.

These relationships were also confirmed in the recent experimental studies by Fujii et al. (2009) and Sakakiyama et al. (2010). Thus, they are thought to be common characteristics of the tsunamiinduced topography changes.

\section{Acknowledgments}

The bathymetry data near Kirinda port before and after the 2004 Indian Ocean tsunami was provided by the Japan International Cooperation Agency.

\section{REFERENCES}

Fujii, N., M. Ikeno, T. Sakakiyama, M. Matsuyama, M. Takao, and T. Mukouhara. 2009. Hydraulic experiment on flow and topography change in harbor due to tsunami and its numerical simulation, Annual Journal of Coastal Engineering, JSCE, 56, 291-295 (in Japanese).

Fujii, Y., and K. Satake. 2007. Tsunami source of the 2004 Sumatra-Andaman earthquake inferred from tide gauge and satellite data, Bulletin of the Seismological Society of America, 97, S192-S207.

Kihara, N., and M. Matsuyama. 2008. Hydrostatic three dimensional numerical simulations of tsunami scour in harbor, Annual Journal of Coastal Engineering, JSCE, 54, 516-520 (in Japanese).

Kihara, N., and M. Matsuyama. 2010. Three-dimensional hydrostatic numerical simulations on tsunami-induced sediment transport in a model harbor, Proceedings of $3^{\text {rd }}$ International Tsunami Field Symposium, 31-32.

Mellor, G.L., and T. Yamada. 1982. Development of a turbulence closure model for geophysical fluid problems, Review of Geophysical and Space Physics, 20, 851-875. 
Nishihata, T., Y. Tajima, Y. Moriya, and T. Sekimoto. 2006. Topography change due to the Dec 2004 Indian Ocean Tsunami - Field and numerical study at Kirinda port, Sri Lanka, Proceedings of $30^{\text {th }}$ International Conference on Coastal Engineering, ASCE, 1456-1468.

Ohie, T., S. Koshimura, H. Yanagisawa, and F. Imamura. 2006. Numerical modeling of the 2004 Indian Ocean Tsunami and damage assessment in Banda Aceh, Indonesia, Annual Journal of Coastal Engineering, JSCE, 53, 221-225 (in Japanese).

Sakakiyama, T., M. Matsuyama, and T. Yoshii. 2010. Experimental study on bottom topography change in harbor due to tsunami, Proceedings of $32^{\text {nd }}$ International Conference on Coastal Engineering, ASCE, (in prepared).

Takahashi, J., K. Goto, T. Oie, H. Yanagisawa, and F. Imamura. 2008. Inundation and topographic change due to the 2004 Indian Ocean Tsunami at the Kirinda port, Sri Lanka, Annual Journal of Coastal Engineering, JSCE, 55, 251-255 (in Japanese).

Takahashi, T., N. Shuto, F. Imamura, and D. Asai. 1999. A movable bed model for tsunamis with exchange rate between bed load layer and suspended layer, Annual Journal of Coastal Engineering, JSCE, 46, 606-610 (in Japanese).

Tomita, T., T. Arikawa, T. Yasuda, F. Imamura, Y. Kawata. 2005. Field survey around south west coast of Sri Lanka of the December 26, 2004 earthquake tsunami disaster of Indian Ocean, Annual Journal of Coastal Engineering, JSCE, 52, 1406-1410 (in Japanese).

Van Rijn, L.C. 1984 (a). Sediment transport, part I: Bed load transport, Journal of Hydraulic Engineering, ASCE, 110, 1431-1456.

Van Rijn, L.C. 1984 (b). Sediment transport, part II: Suspended load transport, Journal of Hydraulic Engineering, ASCE, 110, 1613-1641. 